\title{
PRECISION VALIDATION OF GEANT4 ELECTROMAGNETIC PHYSICS
}

\author{
S. Guatelli, B. Mascialino, M. G. Pia, M. Piergentili \\ INFN - Sezione di Genova \\ Via Dodecaneso 33, 16146 Genova (Italy) \\ Susanna.Guatelli@ge.infn.it; Barbara.Mascialino@ge.infn.it, \\ MariaGrazia.Pia@ge.infn.it; Michela.Piergentili@ge.infn.it \\ L. Pandola, S. Parlati \\ INFN - Laboratori Nazionali del Gran Sasso \\ Strada Statale 17bis del Gran Sasso, 67010 Assergi L'Aquila (Italy) \\ Luciano.Pandola@lngs.infn.it; Sandra.Parlati@lngs.infn.it, \\ K. Amako, K. Murakami, T. Sasaki \\ KEK \\ 1-1 Oho, Tsukuba Ibaraki (Japan) \\ Katsuya.Amako@kek.jp; Koichi.Murakami@kek.jp; Takashi.Sasaki@kek.jp \\ L. Urban \\ Kfki Research Institute for Particle and Nuclear Physics \\ Konkoly Thege Miklos ut 29-33, H-1121 Budapest (Hungary) \\ Laszlo.Urban@cern.ch \\ M. Maire \\ LAPP \\ Annecy (France) \\ Michel.Maire@cern.ch \\ V. Ivanchenko \\ Novosibirsk Budker Institute \\ Prospekt Lavrent'eva 11, 630090 Novosibirsk (Russia) \\ Vladimir.Ivantchenko@cern.ch
}

\begin{abstract}
The Geant4 Simulation Toolkit provides an ample set of physics models to handle the electromagnetic interactions of leptons, photons, charged hadrons and ions.

Because of the critical role often played by simulation in the experimental design and physics analysis, an accurate validation of the physics models implemented in Geant 4 is essential, down to the quantitative understanding of the accuracy of their microscopic features.

results from a series of detailed tests with respect to well established reference data sources and experiments are presented, focusing in particular on the precision validation of the microscopic components of Geant 4 physics, such as cross sections and angular distributions, provided in the various alternative physics models of Geant4 electromagnetic packages. The validation of Geant 4 physics is performed by means of quantitative evaluations of the comparison of Geant 4 models to
\end{abstract}


reference data and well established sources, such as NIST, making use of statistical analysis

algorithms to estimate the compatibility of simulated and experimental distributions.

Key Words: Geant4, Electromagnetic Physics, Validation, NIST

\section{INTRODUCTION}

Geant4 is an Object-Oriented Toolkit [1] for the simulation of the passage of particles through matter.

Geant 4 offers an ample set of complementary and alternative physics models for electromagnetic and hadronic interactions based on either theory, experimental data or parameterisations.

The validation of Geant 4 physics models with respect to experimental data and evaluated references is a critical issue, fundamental to establish the reliability of Geant 4 based simulation.

This paper presents the results of a comparison of Geant 4 electromagnetic models with respect to the reference data of the International Commission on Radiation Units and Measurements (ICRU) [2] and of the National Institute of Standards and Technologies (NIST) [3]. The comparison tests concern the electromagnetic processes of photons, electrons, protons and $\alpha$ particles; they include the total photon attenuation coefficients, the cross sections of the individual processes of photons, stopping power and continuously slowing down approximation (CSDA) range of electrons, protons and $\alpha$ particles in a set of selected materials.

\section{GEANT4 ELECTROMAGNETIC PHYSICS PACKAGES}

The Geant4 electromagnetic packages handle the electromagnetic interactions of leptons, photons, hadrons, ions and muons. They include models for multiple scattering, ionization, Bremsstrahlung, positron annihilation, photoelectric effect, Compton and Rayleigh scattering, gamma conversion, synchrotron and transition radiation, Cherenkov effect, refraction, reflection, absorbtion, scintillation, fluorescence, Auger effect, Particle Induced X-ray Emission (PIXE) [1].

In this study we present tests concerning the Standard and Low Energy electromagnetic packages.

\subsection{Standard Package}

The Standard Package [1] [4] [5] provides a variety of models based on an analytical approach to describe the interactions of electrons, positrons, photons, and charged hadrons in the energy range between $1 . \mathrm{keV}$ and $100 . \mathrm{TeV}$. The models assume that the atomic electrons are quasi-free (i.e., their binding energy is neglected except for photoelectric effect), while the atomic nucleus is fixed (i.e., the recoil momentum is neglected).

\subsection{Low Energy Package: evaluated data driven approach}

The Low Energy Electromagnetic Package [1], [10] extends the range of coverage of electromagnetic interactions in Geant4 down to lower energy than the Standard Package. This Low Energy Package approach exploits evaluated data libraries (EPDL97 [6], EEDL [7] and EADL [8]) that provide data for the calculation of the cross-sections and the sampling of the final 
state for the modeling of photon and electron interactions with matter. The current implementation of low energy electron and photon processes can be used down to 250 . eV. The Low Energy Package handles the ionization by hadrons and ions [11], [12]. It adopts different models depending on the energy range and the particle charge. In the high energy domain $(>2$. $\mathrm{MeV})$ the Bethe-Bloch formula, while in the low energy one $(<1 . \mathrm{keV}$ for protons $)$ the free electron model are applied respectively. In the intermediate energy range parameterized models based on experimental data from the Ziegler [13] and ICRU [2] reviews are implemented; corrections due to the molecular structure of materials and the effect of the nuclear stopping power are taken into account.

The physics models originally developed for the Penelope Simulation Toolkit [9] have been re-engineered in Geant 4 as an alternative and independent set of processes in the Low Energy Package. These models are based on an approach which combines numerical data with analytical cross section models for the different interaction mechanisms. They include low-energy and atomic effects (e.g. Doppler broadening, shell effects) and can be applied to energies from a few hundred $\mathrm{eV}$ to about $1 . \mathrm{GeV}$.

\section{EXPERIMENTAL SET-UP OF THE TESTS}

The experimental set-up was specific to the the physics quantity object of the test, and it reproduced the same experimental conditions of the reference data.

In each test, the behavior of the electromagnetic models in a selected group of materials was evaluated: beryllium, aluminum, silicon, iron, germanium, silver, gold, lead, uranium and water. Materials cover the entire periodic table of the elements in terms of the atomic number $\mathrm{Z}$. The behavior of particles in water was studied as well because of the importance of this material in medical physics applications.

\subsection{Photon attenuation coefficients}

Monochromatic photon beams impinge on a slab of a chosen material. The result of the test is the attenuation coefficient $\frac{\mu}{\rho}$, calculated as follows:

$$
\frac{\mu}{\rho}=-\frac{1}{d \rho} \ln \left(\frac{N}{N_{0}}\right),
$$

where $\rho$ is the density of the target material, $d$ is the thickness of the slab along the photon incident direction, $N_{0}$ is the number of incident monochromatic photons, $N$ is the number of photons emerging from the target without interacting.

The photon total attenuation coefficients have been calculated activating the photoelectric effect, the Compton effect, the pair production. In the case of testing the Low Energy Package, the Rayleigh effect is also activated*.

\subsection{Stopping power and CSDA range}

The stopping power and the CSDA range have been calculated for electrons, protons and $\alpha$ particles.

*The Rayleigh effect is not available in the Geant4 Standard electromagnetic physics Package. 
The experimental conditions are the same for the three types of particles. Each type of particle is generated with a chosen energy from the center of a box, filled with one of the materials listed above. The multiple scattering and the energy loss fluctuations are not active, according to the stopping power and CSDA range definitions.

\section{REFERENCE DATA}

The reference data adopted are ICRU and NIST. The XCOM database reports the photon cross sections and attenuation coefficients. The sum of the interaction coefficients for the individual processes is equal to the total attenuation coefficient. This database comprehends all the elements of the periodic table over a wide range of energies. The XCOM database derives from references: [14], [15], [16], [17].

ESTAR generates stopping powers and CSDA ranges for electrons which are the same as those tabulated in ICRU Report 37 [18], while PSTAR and ASTAR generate the stopping powers and CSDA ranges for protons and $\alpha$ particles on the basis of ICRU Report 49 [19].

\section{STATISTICAL ANALYSIS}

Comparisons between reference data and Geant 4 simulations have been performed by means of a Goodness-of-Fit Statistical Toolkit [22], specialised in the comparison of data distributions.

For any physical quantity of interest and for any element considered, the aim of the comparison was to test whether the physical quantity obtained with Geant4 specific electromagnetic Packages agreed with reference data all over the range of energies E. Table I summarises the specific models activated for the particles considered.

Among the ones available in the Goodness-of-Fit Statistical Toolkit, we selected the Chi-squared test, as this is the only Goodness-of-Fit algorithm including data uncertainties in the computation of the test statistics. The Goodness-of-Fit Statistical Toolkit returned the computed Chi-squared value together with the number of degrees of freedom and the p-value of the comparison. The p-value represents the probability that the test statistics has a value at least as extreme as that observed, assuming that the null hypothesis is true. P-values higher than the confidence level $\alpha=0.05$ set a priori led to the acceptance of the null hypothesis, stating the equivalence between reference data and Geant4 simulations.

\section{RESULTS}

For all the physical quantities considered, Geant 4 is able to reproduce with high accuracy the reference data with any of the electromagnetic models considered. As an example, a few of the results of the test are shown in the following figures:

- Fig. 1 shows the X-ray total attenuation coefficient in uranium,

- Fig. 2 represents the CSDA range of electrons in lead,

- Fig. 3 represents the stopping power of protons in aluminum,

- Fig. 4 represents the stopping power of $\alpha$ particles in silver. 
The Goodness-of-Fit tests performed for all the selected elements demonstrate that all the Geant 4 electromagnetic packages can simulate with great accuracy all the specific physical quantities considered in the whole energy range selected. All the statistical comparisons, in fact, led to the acceptance of the null hypothesis, stating that there is not a statistical difference between reference data and the specific Geant 4 simulations. Fig. 5, 6 and 7 represent three examples of the Goodness-of-Fit test results: the p-values (respectively of the total attenuation coefficient, electron stopping power and proton stopping power tests) are plotted as a function of the atomic number $\mathrm{Z}$ of the selected elements. In any case, it was obtained a p-value higher than the confidence level set. This led to the acceptance of the null hypothesis, stating that there is no difference between reference data and Geant 4 simulations. The oscillations of the p-values as a function of the atomic number $\mathrm{Z}$ are ascribable to the differences of the physics models contained in Geant 4 .

\section{CRITICAL DISCUSSION}

\subsection{Photons}

- The three Geant4 models reproduce total attenuation coefficients with high accuracy, the two Low Energy approaches exhibiting the best agreement with reference data.

- Concerning the photoelectric cross section, the three Geant4 models reproduce reference data with high accuracy; the two Geant4 Low Energy models exhibit the best agreement.

- Both Compton scattering and pair production cross sections are reproduced with high accuracy by the three Geant 4 models; the Geant4 Low Energy EPDL model exhibits the best agreement with reference data.

- In the case of Rayleigh scattering cross sections, the Geant4 models seemed to be in disagreement with the reference data for some materials. The disagreement between NIST reference data and data coming from the recent library EPDL97 within the range of energies between $1 . \mathrm{keV}$ and 1 . MeV has been already underlined and discussed in a recent paper [23]. In his paper the author concluded that EPDL97 [6] is the most up-dated, complete and consistent data library available at the moment. As shown in Fig. 8 in the case of a gold slab, Rayleigh cross section from XCOM and EPDL present major deviations. The results obtained with the Geant4 Low Energy EPDL approach reproduce the EPDL original data.

\subsection{Electrons}

The validation test exhibited that all the three Geant 4 physics models are in excellent agreement with the NIST reference data; the test has not pointed out any particular difference among the three approaches.

\subsection{Protons and $\alpha$ particles}

The Geant4 Low Energy Package contains ICRU 49 parameterisation as one of its models. In this case the comparison between Geant 4 simulations and reference data is to be considered as a verification, rather than as a validation. The test showed some differences between the Low Energy Package based on Ziegler parametrisations and ICRU/NIST data; however the Ziegler models represent an authoritative reference in this physics domain, of comparable relevance as 
the ICRU 49 reference; in this case the comparison between the models should be retained for its intrinsic interest, but it should not be considered as the validation of one with respect to the other.

As expected, the Low Energy Package shows a more precise agreement with respect to the Standard Package in the lower energy range $(\mathrm{E} ; 2 . \mathrm{MeV})$.

\section{CONCLUSIONS}

A systematic test has been performed comparing all the Geant4 simulation models for electrons, photon, protons and $\alpha$ particles with respect to the ICRU and NIST references. All the models for electrons, photons and protons are found in good agreement with the reference data; for $\alpha$ particles the Geant4 Low Energy Package exhibits a good agreement over the whole energy range, while the Standard Package is in agreement with the reference for energies above 0.05 $\mathrm{MeV}$.

A quantitative statistical analysis has allowed to point out the respective strengths of the various Geant 4 models in detail, for each of the physics distributions reported in the ICRU and NIST references.

\section{REFERENCES}

1. Geant4 Collaboration, “Geant4 - a simulation toolkit,” NIM A, 506, pp. 250-303 (2003).

2. A. Allisy et al., "ICRU Report 49," (1993).

3. "NIST web page," http://www.nist.gov (2002).

4. H. Burkhardt, V. M. Grichine, P. Gumplinger, V. N. Ivanchenko, R. P. Kokoulin, M. Maire, L. Urban, "Geant4 Standard Electromagnetic Package for HEP Applications," Proceeding of CHEP 2004, Interlaken (Switzerland), September 2004 (2004).

5. A. G. Bogdanov, H. Burkhardt, V. N. Ivanchenko, S. R. Kelner, R. P. Kokoulin, M. Maire, A. M. Rybin, L. Urban, "Geant4 Simulation of High Energy Muon Interactions," Proceeding of CHEP 2004, Roma (Italy), October 2004, (2004).

6. D. Cullen, J. H. Hubbell, L. Kissel, “The Evaluated Photon Data Library, '97 version,” UCRL-50400, Vol. 6, Rev. 5 (1997).

7. S. T. Perkins, D. E. Cullen, S. M. Seltzer, "Tables and Graphs of Electron-Interaction Cross-Sections from $10 \mathrm{eV}$ to $100 \mathrm{GeV}$ Derived from the LLNL Evaluated Electron Data Library (EEDL), Z=1-100," UCRL-50400, Vol. 31.

8. S. T. Perkins, D. E. Cullen, M. H. Chen, J. H. Hubbell, J. Rathkopf, J. Scofield, "Tables and Graphs of Atomic Subshell and Relaxation Data Derived from the LLNL Evaluated Atomic Data Library (EADL), Z=1-100," UCRL-50400, Vol. 30.

9. J. Sempau, J. M. Fernandez-Varea, E. Acosta, F. Salvat, "Penelope - A code system for Monte Carlo simulation of electron and photon transport," Workshop Proceeding Issy-les-Moulineaux, Issy-les-Moulineaux (France) (2001).

10. S. Chauvie, G. Depaola, V. Ivanchenko, F. Longo, P. Nieminen, M. G. Pia, "Geant4 Low Energy Electromagnetic Physics," Proceeding of CHEP 2001, Beijing (China), September 2001, pp. 337-340 (2001). 
11. S. Giani et al., "CERN-OPEN-99-121,” (1999).

12. S. Giani et al., "CERN-OPEN-99-300,” (1999).

13. H. H. Andersen, J. F. Ziegler, The Stopping and Ranges of Ions in Matter, Pergamon Press, Oxford \& United Kingdom (1997).

14. J. H. Hubbell, "Photon Mass Attenuation and Energy Absorption Coefficients from $1 \mathrm{keV}$ to 20 MeV,” Int. J. Appl. RadiatIsotopes, 33, pp. 1269-1290 (1982).

15. J. H. Hubbell, W. J. Veigele, E. A. Briggs, R. T. Brown, D. T. Cromer, R. J. Howerton "Atomic Form Factors, Incoherent Scattering Functions, and Photon Scattering Cross Sections," J. Phys. Chem. Ref. Data, 4, pp. 471-538 (1975).

16. J. H. Hubbell, Overbo "Relativistic Atomic Form Factors and Photon Coherent Scattering Cross Sections,” J. Phys. Chem. Ref. Data, 8, pp. 69-105 (1979).

17. J. Leroux, T. P. Thinh, Revised Tables of X-ray Mass Attenuation Coefficients, Corporation Scientifique Classique, Quebec \& Canada (1977).

18. ICRU International Commission on Radiation Units and Measurements. "ICRU Report 37, Stopping Powers for Electrons and Positrons", 1984.

19. ICRU International Commission on Radiation Units and Measurements. "ICRU Report 49, Stopping Powers and Ranges for Protons and Alpha Particles", 1993.

20. H. H. Andersen, J. F. Ziegler, Hydrogen: Stopping Powers and Ranges in All Elements. Vol. 3 of The Stopping and Ranges of Ions in Matter, Pergamon Press, Elmsford \& New York (1977).

21. J. F. Ziegler, Helium: Stopping Powers and Ranges in all Elemental Matter. Vol. 4 of The Stopping and Ranges of Ions in Matter, Pergamon Press, Elmsford \& New York (197).

22. G. A. P. Cirrone, S. Donadio, S. Guatelli, A. Mantero, B. Mascialino, S. Parlati, M. G. Pia, A. Pfeiffer, A. Ribon, P. Viarengo, “A Goodness-of-Fit Statistical Toolkit,” IEEE-Transactions on Nuclear Science, 51 (5), pp. 2056-2063 (2004).

23. H. Zaidi, "Comparative evaluation of photon cross section libraries for materials of interest in PET Monte Carlo simulation,” IEEE Transaction on Nuclear Science, 47, pp. 2722-2735 (2000). 
Table I: Geant4 electromagnetic models under test.

\begin{tabular}{|l|l|}
\hline Particle & \multicolumn{1}{|c|}{ Geant4 Models } \\
\hline Photon & $\begin{array}{l}\text { Geant4 Low Energy - EPDL } \\
\text { Geant4 Low Energy - Penelope } \\
\text { Geant4 Standard }\end{array}$ \\
\hline Electron & $\begin{array}{l}\text { Geant4 Low Energy - EEDL } \\
\text { Geant4 Low Energy - Penelope } \\
\text { Geant4 Standard }\end{array}$ \\
\hline Proton & $\begin{array}{l}\text { Geant4 Low Energy - ICRU 49 } \\
\text { Geant4 Low Energy - ZIEGLER 1985 } \\
\text { Geant4 Low Energy - ZIEGLER 2000 } \\
\text { Geant4 Standard }\end{array}$ \\
\hline$\alpha$ particle & $\begin{array}{l}\text { Geant4 Low Energy - ICRU 49 } \\
\text { Geant4 Low Energy - ZIEGLER 1977 } \\
\text { Geant4 Standard }\end{array}$ \\
\hline
\end{tabular}

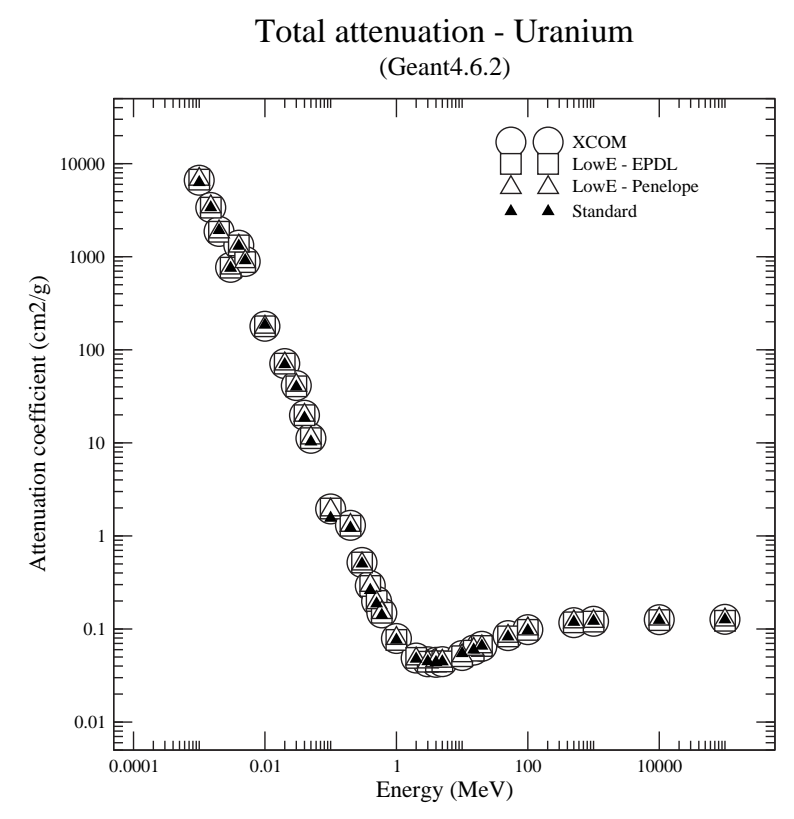

Figure 1: Total attenuation coefficient in uranium as a function of the photon incident energy. 


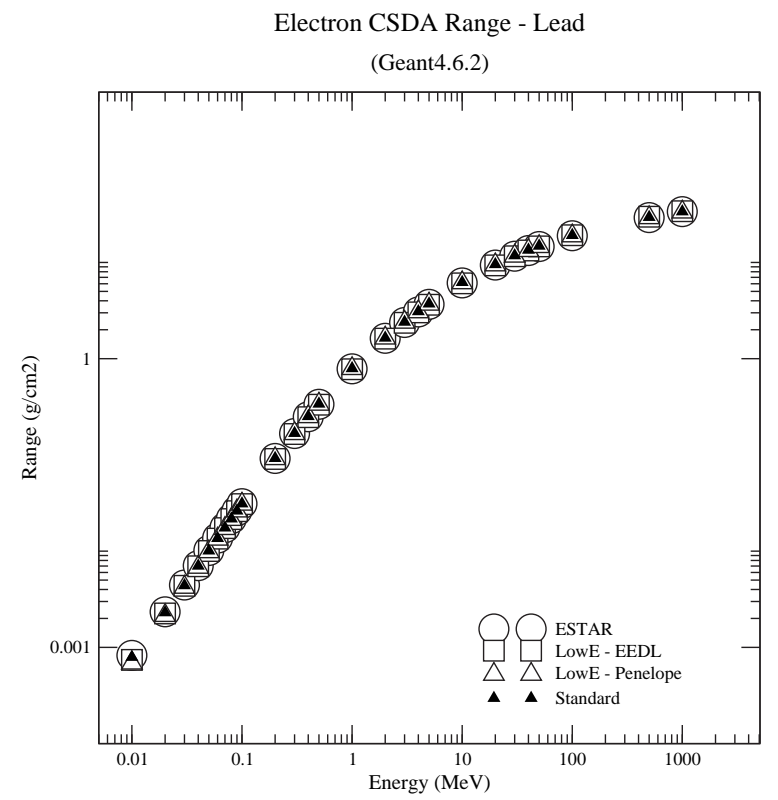

Figure 2: Electron CSDA range in lead as a function of the electron incident energy.

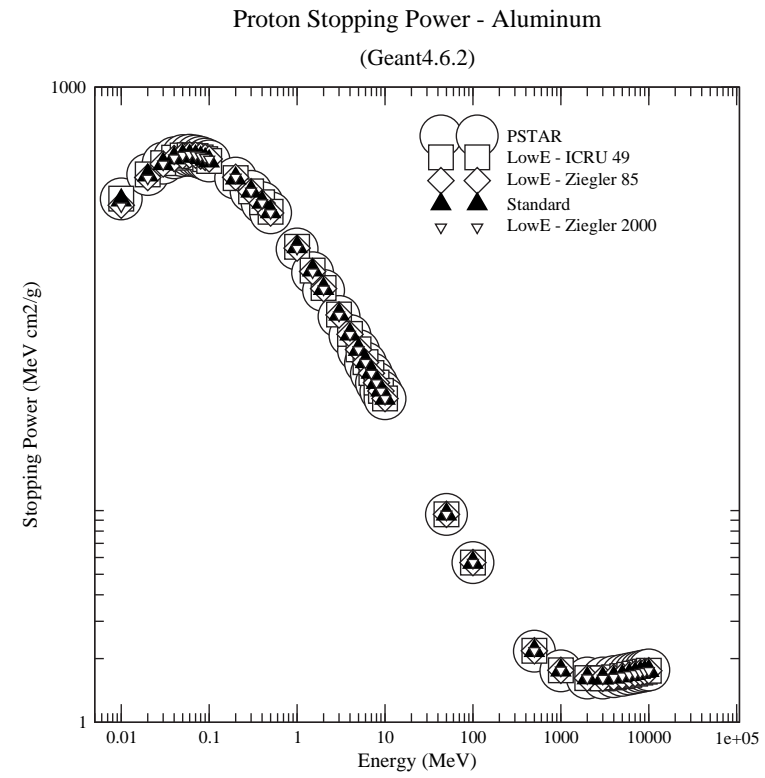

Figure 3: Proton stopping power in aluminum as a function of the proton incident energy. 


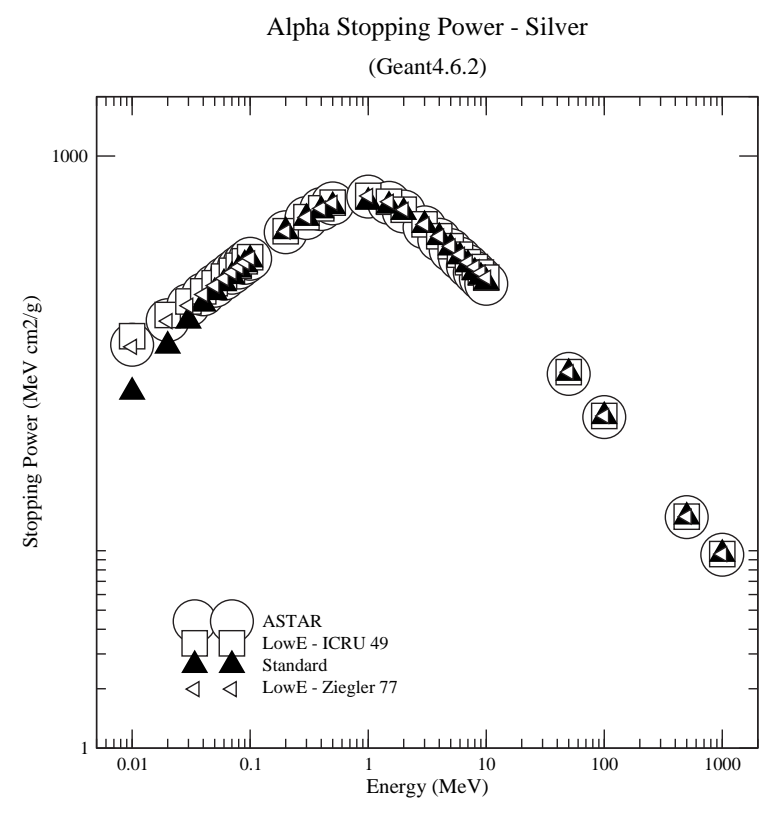

Figure 4: $\alpha$ stopping power in silver as a function of the $\alpha$ incident energy.

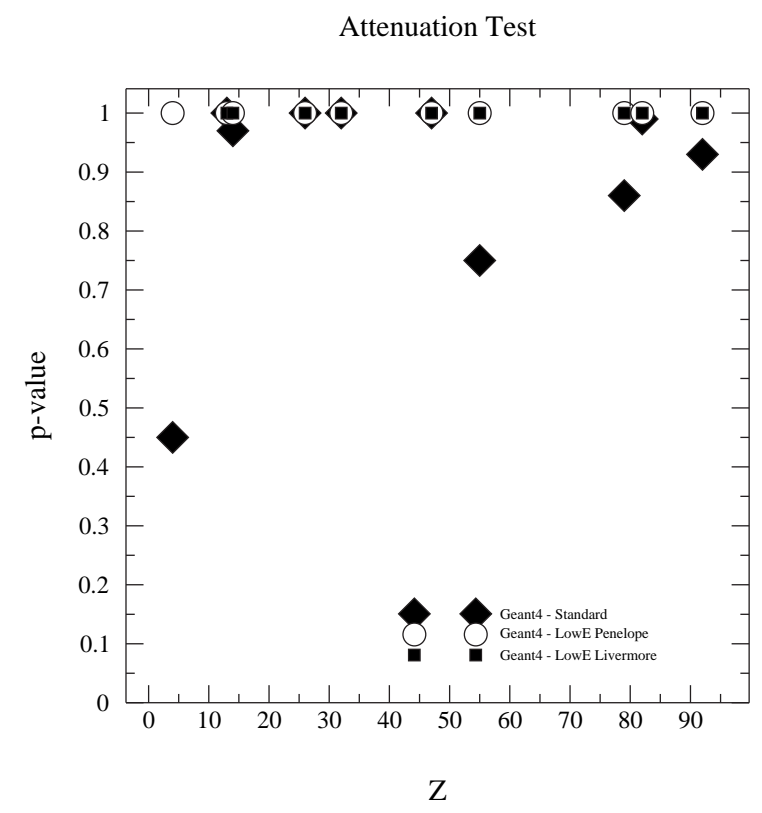

Figure 5: Goodness-of-Fit test results concerning the total attenuation coefficient plotted as a function of the atomic number $Z$ of the selected elements (Standard: black diamonds, Low Energy EPDL: black squares, Low Energy Penelope approach: empty circles). All the p-values obtained are higher than the confidence level set $(\alpha=0.05)$. 
Electron Stopping Power Test

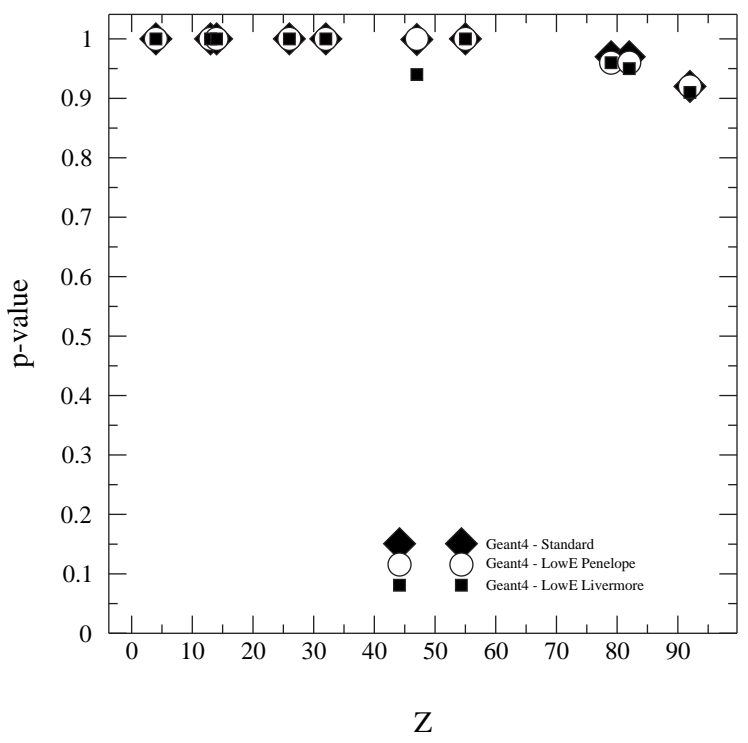

Figure 6: Goodness-of-Fit test results concerning the electron stopping power plotted as a function of the atomic number $Z$ of the selected elements (Standard: black diamonds, Low Energy EPDL: black squares, Low Energy Penelope approach: empty circles). All the p-values obtained are higher than the confidence level set $(\alpha=0.05)$. 
Proton Stopping Power Test

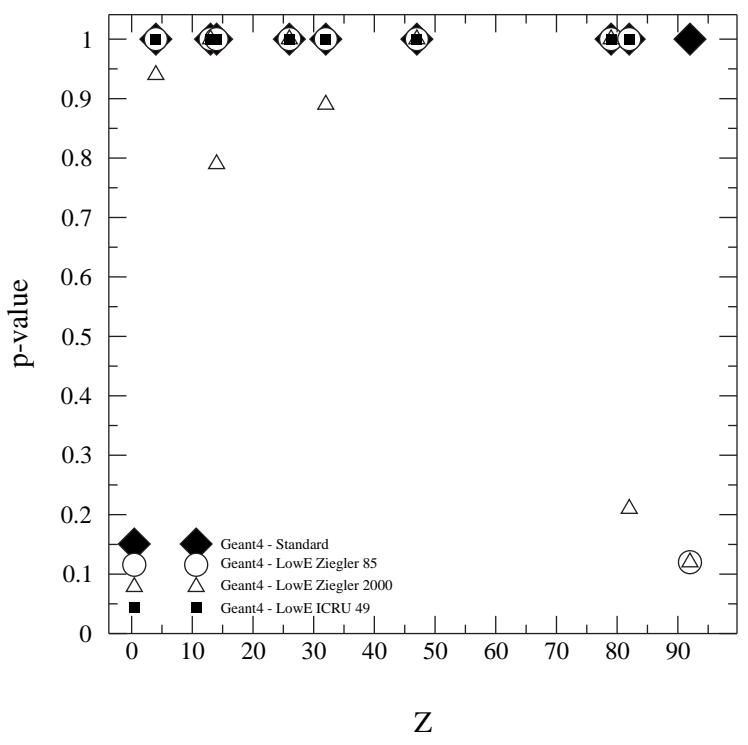

Figure 7: Goodness-of-Fit test results concerning the proton stopping power plotted as a function of the atomic number $Z$ of the selected elements (Standard: black diamonds, Low Energy ICRU 49: black squares, Low Energy Ziegler 1985: empty circles, Low Energy Ziegler 2000: empty triangles). All the $\mathbf{p}$-values obtained are higher than the confidence level set $(\alpha=0.05)$. 
Coherent Scattering - Gold

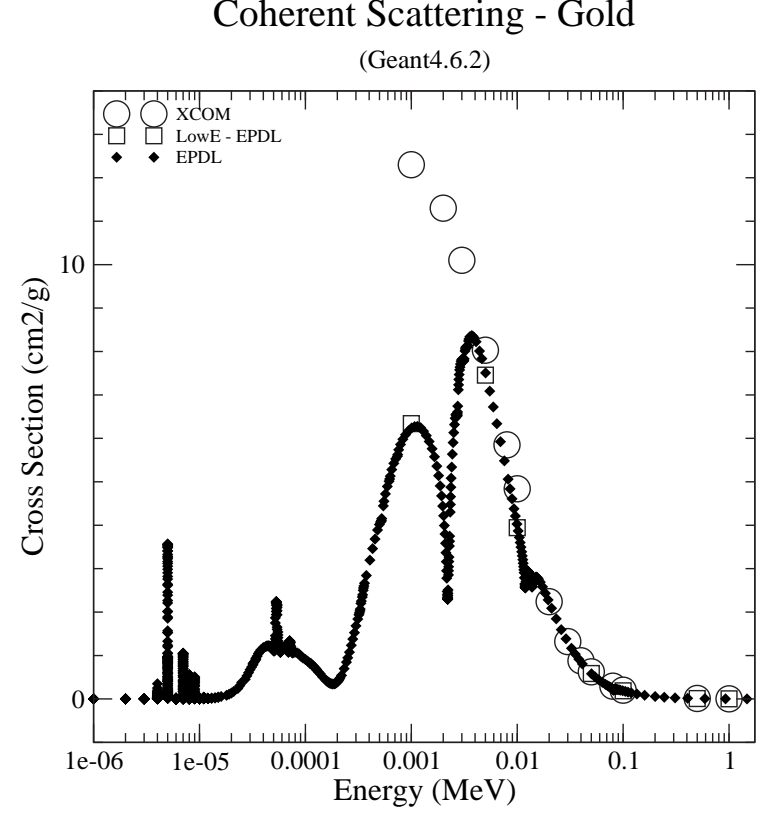

Figure 8: Comparison between Rayleigh cross section from XCOM (black circles) and EPDL (white circles) in the specific case of a gold slab. Note the major deviations between the two data sets. The results obtained with the Geant4 Low Energy Package (black triangles) are in perfect agreement with the EPDL data; this is meant to be a verification of the Geant 4 simulation dedicated to this specific test. For more details see text. 